\title{
Computational Modeling of Coupled Free and Porous Media Flow for Membrane-based Filtration Systems: A Review
}

\author{
Antonios Parasyris ${ }^{\mathrm{a}}$, Christopher Brady ${ }^{\mathrm{b}}$, Diganta B. Das ${ }^{\mathrm{b} *} \&$ Marco Discacciati $^{\mathrm{a}}$ \\ aDepartment of Mathematical Sciences, ${ }^{\mathrm{b}}$ Chemical Engineering Department, \\ Loughborough University, Epinal Way, Loughborough LE11 3TU, UK
}

Submitted: 15/03/2019. Revised edition: 28/07/2019. Accepted: 13/08/2019. Available online: 20/09/2019

\begin{abstract}
We review different mathematical models proposed in literature to describe fluid-dynamic aspects in membrane-based water filtration systems. Firstly, we discuss the societal impact of water filtration, especially in the context of developing countries under emergency situations, and then review the basic concepts of membrane science that are necessary for a mathematical description of a filtration system. Secondly, we categorize the mathematical models available in the literature as (a) microscopic, if the pore-scale geometry of the membrane is accounted for; (b) reduced, if the membrane is treated as a geometrically lowerdimensional entity due to its small thickness compared to the free flow domain; (c) mesoscopic, if the characteristic geometrical dimension of the free flow domain and the porous domain is the same, and a multi-physics problem involving both incompressible fluid flow and porous media flow is considered. Implementation aspects of mesoscopic models in CFD software are also discussed with the help of relevant examples.
\end{abstract}

Keywords: Coupled free and porous-media flows, membrane filtration, CFD, coupling conditions

\subsection{INTRODUCTION}

\subsection{Societal Needs}

Humans require 3-5 liters of clean and safe drinking water every day in order to survive [1]. Man-made and natural disasters can jeopardize this by damaging water supply infrastructure, putting lives at risk through the difficulty of accessing clean and safe drinking water [2, 3]. Many first-world countries have systems and infrastructure in place to effectively react to disasters and emergency situations; however, this is not the case for all developing countries [4]. The UN has demonstrated the importance of solving this humanitarian issue through its sixth UN Sustainable Development Goal, which concerns the access to clean and safe drinking water. A possible solution to address this goal is an affordable and effective water purification system for emergency use in developing countries. A point-of-use water treatment (PoUWT) filtration system can potentially fulfill these requirements by offering a device that effectively supplies a temporary source of clean and safe drinking water until outside aid is received [5].

Membrane processes such as microfiltration (MF) and ultra-filtration (UF) have been widely used for water treatment in recent years $[6,1]$. They have been used as alternative technologies to conventional methods such as coagulation, sedimentation, ozonation, granular activated carbon, flocculation/chlorination, slow sand filtration, etc. The reason for this is 
that membrane processes are cost effective, require simple conditions for operation and have a high output, all with a lower energy consumption and chemical use.

Extensive research has been invested into determining possible ways of purifying contaminated water with low cost/maintenance devices that use little to no chemicals, minimizing possible harmful side effects. The success of the proposed devices lies heavily on social acceptance, which is mainly dependent on scientifically proven efficiency achieved through optimization. This is why some authors conduct experimental studies, for example [7] for an ultra-low pressure with dead-end ultrafiltration without backflushing and cleaning.

However, experimentation is limited due to the costs of constructing multiple configurations of the same device whilst having trained staff conduct many trial- and-error experiments to determine how to maximize flux and minimize blocking effects for each device. Then, extensive comparison with other types of devices must be carried out to validate a specific approach, as, e.g., [5] did for a hybrid circular flow and a stirred dead-end system.

To overcome this challenge, computational fluid dynamics (CFD) models are employed to simulate the operation of any configuration, and determine the fluid velocity and pressure at any given point, thus making it possible to estimate the outward flux of the device, and hence maximize it by changing certain geometrical or membrane-specific parameters.

This literature review presents different mathematical and computational approaches to effectively and reliably model the process of filtration of an incompressible fluid through a porous material with application to membrane-based water purification. More precisely, differently from other review papers focused only on fluiddynamic aspects either in the feed domain or inside the membrane, here we consider the whole filtration process considering both the feed domain the porous materials typically used for ultra- and micro-filtration.

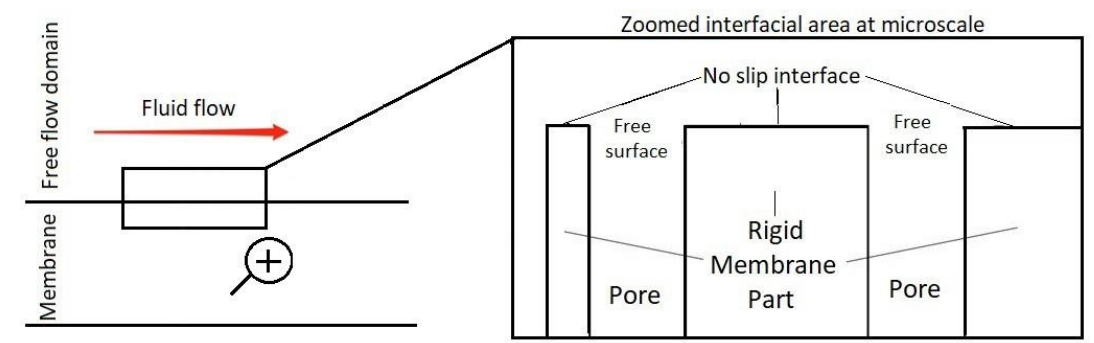

Figure 1 Free flow/porous medium (membrane) setting (left) and zoomed interface area at the micro-scale (right)

\subsection{Coupled Free and Porous Flow}

The difficulty in modeling membranebased filtration devices arises from the fact that the porous domain has a very complicated microstructure almost impossible to represent, and that in comparison with the free flow domain, the porous medium has usually very small thickness. The complicated geometry inside the porous medium is designed to allow flow through micropores whilst blocking impurities above a certain size threshold. This geometry is too complicated to model exactly, which makes the problem 
computationally intractable. Not only one should represent the microscopic channels inside the porous material but, at the interface with the feed domain, one should also treat each pore as a free flow domain and each rigid part of the membrane as a no-slip surface (see Figure 1). To avoid this computational complexity and cost, averaged equations are used to determine the fluid flow behavior in the porous medium. This approach does not come without disadvantages as one needs to determine which equations should be solved in each domain, the coupling conditions to describe the flow behavior for the common free porous medium interface, and the exact location of the latter. We will discuss these aspects in the paper that is organized as follows. In Section 2, we review basic membrane characteristics required in the rest of the paper. Section 3 presents the differential models for the accurate representation of a filtration device. We categorize the models found in literature and then focus on the mesoscopic flow models which are based either on the Navier-Stokes equations coupled with either Darcy's law or Brinkman equation, or on the socalled one-domain model. We discuss coupling conditions for heterogeneous models and the boundary conditions that should be imposed on a simple hybrid filtration geometry. Finally, Section 4 is devoted to the numerical approximation and solution of the coupled heterogeneous NavierStokes/Darcy model using CFD software.

\subsection{MEMBRANE CHARACTERISTIC}

\subsection{Types of Membranes}

Systems that incorporate semipermeable membranes for filtration are an active field of both experimental and theoretical research due to their practical significance and increasing number of applications. Some examples are shown in [8]. Water treatment processes employ several types of membranes based on the target particle size to be removed and the required operating pressure. They include micro-filtration (MF), ultrafiltration (UF), nano-filtration (NF) and reverse osmosis (RO), which are intended for particles with the following sizes: $\mathrm{MF}$ : $0.1-1 \mu \mathrm{m}$, UF: $0.005-2 \mu m, \mathrm{NF}:>0.002 \mu m$, RO: most commonly used for desalination with high pressures. Applications of membrane filtration in water treatment can be divided into two groups. Firstly, MF and UF for the removal of particulate material and microorganisms and secondly, NF and RO for the removal of dissolved material and micro-pollutants based on their molecular cutoff.

\subsection{Membrane Modules}

Membrane modules consist of the membrane, a pressure support structure, a feed inlet, the permeate outlet, retentate streams and the overall support system. There are five main types of modules: plate-and-frame, tubular, spiral wound, hollow fiber and flat sheet (see, e.g., [9]). The plateand-frame module is the simplest configuration, consisting of two end plates, the flat sheet membrane, and spacers. In tubular modules, the membrane is often on the inside of a tube, and the feed solution is pumped through the tube. The most popular module in industry for $\mathrm{NF}$ or $\mathrm{RO}$ membranes is the spiral wound module. This module has a flat sheet membrane wrapped around a perforated permeate collection tube. The feed flows on one side of the membrane. Permeate is collected on 
the other side of the membrane and spirals in towards the central collection tube. Hollow fiber modules used for seawater desalination consist of bundles of hollow fibers in a pressure vessel. They can have a shell-side feed configuration where the feed passes along the outside of the fibers and exits the fiber ends. Hollow fiber modules can also be used in a bore-side feed configuration where the feed is circulated through the fibers. Hollow fibers employed for wastewater treatment and in membrane bioreactors are not always used in pressure vessels. Bundles of fibers can be suspended in the feed solution, and the permeate is collected from one end of the fibers. Lastly, whilst the flat sheet membrane is used in other modules, it can also be considered a module itself, as it can be used for MF and UF in low pressure systems for water purification purposes.

\subsection{Operation Modes}

An important categorization of membrane systems is based on their operation mode. There are two main modes, which are based on the flow direction compared to the membrane surface.

Firstly, we have dead-end filtration (most commonly with tubular and flat sheet membranes), where bulk flow is perpendicular to the filter medium [10], and an accumulation of feed particles occurs at the membrane surface. This blocks the membrane surface [11], often leading to cake formation [12], which creates an additional resistance to mass transfer [13, 14], and, hence, a decrease in permeate flux over time [12]. Authors in [15] concluded that fouling phenomena is the limiting factor in membrane performance.

Secondly, we have crossflow filtration, where the feed flows parallel to the membrane surface, and thus, the concentration changes as a function of distance from the inlet [12]. There is a smaller decrease in flux with time compared to dead-end filtration, which is owed to the reduced effect of fouling through tangential flow across the membrane, preventing significant solid deposition [10]. This is the main advantage of crossflow over dead-end filtration.

Some authors propose hybrid systems (see, e.g., $[1,12]$ ) to combine the advantages of both methods, such as higher filtration velocity, the reduction of pore blockages, and concentration polarization. We will give a simple example of such a configuration in Sect. 3.3.1.

\subsection{Fouling}

Fouling is defined as the deposition of matter onto or into a membrane, which causes a change in permeate flux and in the amount of rejected particles [10]. The driving force for membrane filtration in water treatment is the pressure gradient across the membrane, which results in a convective transport of material from the bulk to the membrane surface. Solvent (water) permeates through the membrane and solutes (dissolved and particulate material) are partly or completely retained by the membrane. The retained dissolved solutes and particulate material accumulate in a boundary layer at the membrane surface and a concentration build-up in time, the so-called concentration polarization, is observed. As a result of the creation of the thin concentration boundary layer near the membrane surface, the filtrate flux declines over time [12] and reaches an equilibrium determined by the extent of fouling [10].

There are two types of fouling: internal and external. Internal fouling is when solutes and particles are 
entrapped or adsorbed in or on the internal pore structure of the membrane, which is known to be semipermanent. External fouling is the formation of a cake on the membrane surface, due to the rejection of particles by the membrane, which is known to be reversible through back-washing. Both these two types of fouling cause a resistance to mass transfer; hence it is desirable to minimize the amount of fouling in the system [10]. The resistances to mass transfer can be attributed to pore blocking, adsorption, gel and cake formation, and concentration polarization, which, along with membrane resistance, contribute towards the overall resistance to mass transfer in the system [12].

\subsection{Membrane Surface Morphology}

Factors that might affect modeling in the context of the free/porous interface (see Figure 1) include surface morphology, such as surface roughness and wettability (see, e.g., [16]). Indeed, the membrane surface contains microscopic hills and valleys. We can define the surface roughness with statistical methods that involve data obtained experimentally. As shown in [17], higher values of surface roughness lead to an increased permeate flux and a decreased diffusion path length [18].

Another factor of surface morphology is surface charge. It is desirable for the membrane and particles to have the same charge to promote electrostatic repulsion, reducing fouling. Moreover, hydrophilic (contact angle $<90^{\circ}$ ) membranes are preferred to hydrophobic (contact angle $>90^{\circ}$ ) ones due to the higher free surface energy of the former [1]. Electric effects are beyond the scope of this paper and they will not be considered in the rest of this work.

\subsection{Increased Filtration Efficiency}

To increase efficiency, a significant amount of research is spent on proposing devices that induce artificial fluid flow instabilities like Dean vortices [19, 20] or Taylor bubbles [21], to recirculate the feed. These can be achieved with spacers, rotating machinery, spiral channel systems, stirrers, etc. A good review with experiments on these instabilities is found in [22] and the references therein.

Injecting gas in the feed to create a gas-liquid two-phase crossflow operation and using intermittent slug bubbling through periodic introduction of large bubbles is studied in [23, 24].

The effect of baffles in the context of filtration is studied in [25] for tubular membrane systems, incorporating turbulence effects. This is not common in membrane systems where the Reynolds number generally categorizes flow in the laminar regime, but the obstruction of flow from the baffles and the faster flow regime considered in this study facilitate in the creation of turbulence.

Another wide field of application that is heavily benefited by the CFD modelling is in narrow spacer-filled channels, as the geometric optimization is cumbersome in these complicated configurations. We refer, e.g., to [26] and also to $[27,28,29,30]$ that solve the Navier-Stokes equations in the microscale geometry. The work [31] presents a 3D CFD study on complex spacer geometries as well as a comprehensive literature review on studies that have focused on either optimizing spacer geometric parameters or attempting to gain a better understanding of the mechanisms giving rise to mass transfer enhancement. 


\subsection{CFD MODELING OF MEMBRANE FILTRATION SYSTEMS}

The complexity of membrane based systems for various applications springs mostly from the difficulty of modeling the flux decline due to the impurities that block the pores of the membrane, either internally or sitting on the surface, hindering the membranes' ability to act like a barrier between two domains and to separate a fluid or gas filled with particles. This has been well known for many years: the work of [32] in 1985 is centered around crossflow membrane filtration, and it emphasizes the importance of fluid dynamic behavior to understand concentration polarization and membrane fouling.

CFD simulations offer a deep understanding of flow by reproducing its velocity and pressure field on a discretized version of the geometry. A few difficulties arise when setting up a CFD simulation. Firstly, a complicated geometry must be built, which might be difficult to approximate accurately using a computational mesh. Secondly, a correct set of equations to accurately describe the underlying physics must be selected. These typically involve the balance of forces (momentum equation) and the conservation of mass (e.g., the incompressibility equation). Then, the question of accounting for the porous medium arises, and its coupling with the free flow region. We categorize the coupled models found in literature in three groups.

- Microscopic models: they aim at representing the free/porousmedium system at the microscopic level providing a precise and detailed geometrical description of the membrane.

- Reduced models: upon typically making assumptions on the flow pattern, these models provide a simplified description of the filtration device generally limited to the feed domain.

- Mesoscopic models: they treat the membrane as a uniform porous medium where averaged models, such as Darcy's law, are applied. The fluid is typically described by Navier-Stokes equations leading to a system of partial differential equations.

\subsection{Microscopic Models}

In this framework, the geometry is modeled at the microscale accounting for details that are not seen with the naked eye. In principle, one should represent the whole membrane at microscopic level and then solve fluid equations such as, the Navier-Stokes equations, both inside each of the membrane pores and in the free-fluid region. This approach was used, e.g., in [33], an early study that focused on crossflow filtration over single pores of the membrane. However, this is extremely demanding. Indeed, obtaining a precise representation of the whole membrane requires sophisticated imaging techniques, generating a computational mesh becomes very challenging, and the number of unknowns to describe the velocity and pressure fields, e.g., in a finite element context, make the problem intractable. Some simplifying assumptions can be made to alleviate the computational cost. For example, one can precisely represent only a small portion of the membrane and replicate it to model the whole device assuming that the membrane is uniform and isotropic in each direction. However, due to its computational cost, in practice the microscopic approach is used only to study parts of the filtration device that are of special interest. Examples of real-world applications that this 
approach can tackle are small disturbances in flow using gas bubbles injected in the feed or narrow spacerfilled channels (often in spiral wound membrane modules). We refer the interested reader to $[31,27,28,29$, 30].

Finally, we remark that in the microscopic context coupling different equations is needed only to model impurities in the feed. In the example of spacer-filled geometries, water and solute fluxes are generally described either by diffusion models as seen in [34] where the concentration polarization effects are studied using CFD or by the Spiegler-Kedem model [35].

\subsection{Reduced Models}

To reduce the computational cost of a full micro-scale (and also of a macroscale) simulation, several authors introduced simplifying hypotheses to be able to model the filtration process assuming a certain flow pattern in the feed domain and representing the membrane by ad-hoc boundary conditions instead of describing it by a dedicated model. These approaches led to define reduced models that do not demand a huge computational effort but rely on strong assumptions which make them difficult to adopt in general contexts.

For example, $[36,37]$ assumed that the permeate flux through a membrane was independent of its location on the membrane surface and were able to find an analytic solution for the velocity field in the feed domain. This avoids computing numerically the latter and [38] used the analytic velocity as the convective field for a steady-state convection-diffusion mass transfer equation that was solved by the finite element method for a cross flow polysulphone UF membrane. Thanks to the adopted simplification, for a given permeate flux, [38] could obtain a linear relationship between the diffusion coefficient and the thickness of the concentration boundary layer which was in agreement with the theory and gave indications on how to generate an appropriate finite element mesh near the membrane. Moreover, their finite element analysis could predict the mass transfer coefficient, an important parameter in engineering design and analysis of a membrane filtration process.

Other reduced models consider the membrane as a lower dimensional entity in comparison with the free flow domain so that, e.g., a flat sheet membrane would be considered as a porous wall. This simplifies the equations to be solved, as the presence of the porous medium is simply described by a boundary condition. The basis for this approach was set in [39], where the method is used to model crossflow membrane filtration and the author develops a robust, accurate and cost-effective finite element scheme to simulate the flow in the feed domain under the simplifying assumption that the membrane is regarded as a porous wall. This work was extended in [40] to the case of curved boundaries for crossflow filtration.

Other works represent the presence of the membrane by imposing a no-slip boundary condition for the tangential velocity to the Navier-Stokes equations at a boundary of the feed domain. These works, such as [41, 42], are based on [43] that claims that in crossflow filtration mode, the tangential velocity over the membrane is so low that it can be assumed a noslip surface, and thus impose a zero tangential velocity boundary condition. This can also be seen in [44], where a rectangular reverse osmosis membrane channel is modeled by finite elements and the authors solve the Navier- 
Stokes equations coupled with convection-diffusion equations to describe mass transport with application to salts. Again, the membrane is modelled by a suitable boundary condition that imposes noslip tangential velocity, and a normal velocity according to Darcy's law. The authors also introduce suitable coupling conditions on the membrane for the concentration of the impurities (salts in this case). A similar study for protein ultrafiltration is found in [45].

An attempt to combine the NavierStokes equations and Darcy's law to predict the growth of the concentration polarisation boundary layer in tubular crossflow membrane filters is also found in [46], with special focus on the fluid behavior in a porous tube with variable wall suction. Another similar approach is adopted in [47, 48], where CFD is used to model fluid dynamics gauging, a popular experimental procedure that tracks the build-up and deposition of fouling layers on membrane filtration systems.

In [49], the finite volume method is used to model NF in a slit with a crossflow membrane configuration, solving equations that model the motion and the solute transport of a nonreacting binary solution. The membrane is modeled as a boundary condition, and the results are verified with experimental data. In [50], the membrane is modeled as a solid wall and experimental data are used to develop 2D and 3D models where permeation of solvent molecules is described by appropriate sink terms in the equations of conservation of mass.

The no-slip condition does not apply when rough membrane surfaces and high tangential velocities are present, which usually occurs in operational modes other than crossflow filtration. Also, [51] points out that the slip velocity increases with the size of the membrane pores. Due to this, the zero tangential velocity boundary condition can be replaced by a suitable slip condition similar to the BeaversJoseph relationship [52] that relates the tangential velocity to the tangential shear rate. This involves a slip coefficient that depends on the characteristics of the membrane surface such as its roughness, pore size and structure [53]. For instance, it has been shown that the slip coefficient is higher for a densely packed porous material than for a less densely packed one [54]. The resulting reduced models feature the Navier-Stokes equations in the free flow domain with a permeable boundary condition for the normal flux to describe the presence of the membrane. Work focusing on spiral wound membrane modules is found in [55], where the boundary to represent the membrane is derived from Darcy's law. A more recent study on this approach is [56], where the proposed model is also validated with experimental data, and also [57]. The effect of pulsatile flow in crossflow configurations is studied with the membrane being modeled by a Darcian-like boundary condition. Additionally, a source term is added to the Navier-Stokes momentum equations to account for the permeation through the membrane. This term is again based on Darcy's law and it relates the flux, the transmembrane pressure and the sum of membrane resistance and cake resistance which are estimated from experimental data. An extension of this work is found in [58], where two-phase flow is experimentally and numerically studied in a flat sheet membrane module for concentration polarization in oil-inwater emulsion.

A recent summary of reduced models is found in [59], where the authors review the hydrodynamics of membrane channels, including laminar, turbulent, and transition flow regimes, 
with reference to the effects of osmotic pressure, concentration polarization and cake formation.

A limit case of the reduced modeling approach can be found in [60] which models a laboratory membrane filtration cell operated at low recovery. In this setting, membranes are much thinner than the channel height and are tightly pressed against the lower wall of the channel due to the pressure difference between retentate and permeate sides of the membrane unit. Hence, this study proposes to completely neglect the existence of the membrane in the cell, and instead, treat it as an impermeable no slip wall. However, the authors note the significance of the low recovery assumption for this model to be valid, and that this approach is not realistic for devices with higher recoveries.

\subsection{Mesoscopic Models}

We consider now the mesoscopic approach which can be applied to membrane systems regardless of their operational mode and of any dimensional restrictions, and it is a computationally feasible approach for membrane systems, compared to the microscopic approach. First, we generally describe this approach with reference to the literature, then we formulate it more rigorously for a chosen hybrid operation system to demonstrate its versatility.

On the mesoscale, we treat both the free flow region and the porous medium domain as having the same geometric dimensions. Then, we must specify an appropriate set of equations to be solved in each region as well as suitable coupling conditions between the equations to represent filtration, a controversial subject for which no general consensus seems to exist in the literature. For example, [43] shows that the slip effect at the semi-permeable surface in a crossflow filtration system is practically negligible and thus the no-slip boundary condition can be used, a practice implemented for various applications, for example [41]. A study modelling a 3D multichannel inorganic membrane tube is presented in [61], where the boundary between the free flow and porous domains is said to be a porous jump boundary and a zero tangential velocity condition is imposed along the free flow-porous interface (a technique often seen when modelling crossflow filtration).

The assumption of zero velocity coupling is not known to be true for hybrid configurations, which are more general and have a higher tangential velocity. Also, as already mentioned in Sect. 3.2, this assumption is shown not to be true for membranes with a rough surface, where a slip velocity condition must be imposed, like in [62]. To circumvent the difficulty of choosing coupling conditions, studies like [63] prefer the use of the one-domain approach (see Sect. 3.3.1) as it provides ease of implementation and skips the controversy of the choice of coupling conditions and different representative equations.

With regard to crossflow membrane filtration modelling, a literature review of previous CFD studies is presented in $[64,65]$ together with several computational experiments including curved interfaces and a changing value of the membrane permeability.

In the same spirit of mesoscopic models, [66] offers a novel approach for the coupling of free and porous media flow by defining a viscous transition zone inside the porous domain where the equation used for the free fluid is still valid. In addition, continuity of pressure and velocity is imposed across the whole transition zone. In this way, imposing slip conditions like the Beavers-JosephSaffman condition [52] is unnecessary, 
so that this method only requires estimating the depth of the transition zone.

\subsubsection{Formulation of Mesoscopic Models for a Hybrid Filtration System}

We now focus on formulating more precisely the mesoscopic approach in the case of a hybrid filtration system where no simplifying hypotheses can be made due to the arbitrary nature of the flow.

The membrane is modeled as an isotropic porous medium for simplification. Moreover, we assume that there are no impurities in the feed. The mesoscopic models use systems of partial differential equations to describe flow inside the free flow channel and through the membrane, while they represent the remaining components of the device through suitable boundary conditions. As shown in Figure 2, we denote $\Omega_{f}$ as the free flow domain where the fluid flows above the membrane $\Omega_{p}$. These two regions are non-intersecting and separated by a common surface (interface) $\quad \Gamma_{I}: \Omega_{f} \cap \Omega_{p}=\varnothing, \Gamma_{I}=$ $\overline{\Omega_{f}} \cap \overline{\Omega_{p}}$. The fluid in $\Omega_{f}$ is characterized by its density $\rho$ (e.g., $\rho=$ $10^{-3} \mathrm{~kg} / \mathrm{m}^{3}$ for water) and dynamic viscosity $\mu$ (e.g., $\mu=10^{-3} \mathrm{~Pa} \cdot s$ for water). We indicate $\boldsymbol{u}$ and $p$ as the velocity and pressure of the fluid respectively, and we use the subscripts $f$ or $p$ to indicate if they are considered either in $\Omega_{f}$ or in $\Omega_{p}$. Quantities without any subscript are understood to be defined in both domains.

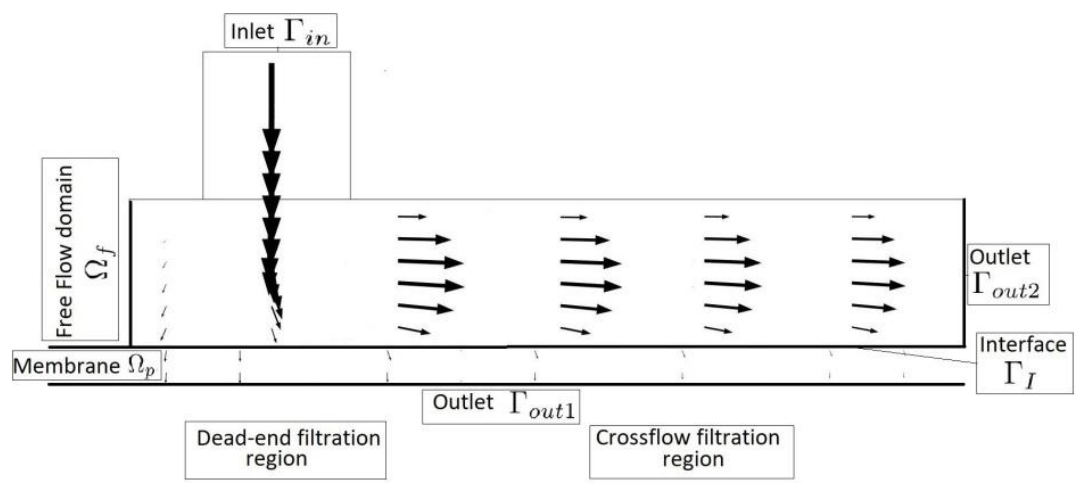

Figure 2 A simple example of a geometrical setting that induces hybrid filtration by combining dead-end and crossflow filtration

Coupled Heterogeneous Models. The first approach we review to model the filtration device is to adopt different sets of equations in the free flow and porous media domains ( $\Omega_{f}$ and $\Omega_{p}$, respectively). These account for the different physics in each region. To solve this coupled system of equations, we need to prescribe suitable sets of coupling conditions across the interface $\Gamma_{I}$.

To be more precise, in $\Omega_{f}$ we consider the incompressible Navier-
Stokes equations:

$$
\begin{gathered}
\rho\left(\boldsymbol{u}_{\boldsymbol{f}} \cdot \nabla\right) \boldsymbol{u}_{\boldsymbol{f}}=\nabla \cdot T\left(\mu ; \boldsymbol{u}_{f}, p_{f}\right)+\rho \boldsymbol{g} \\
\nabla \cdot \boldsymbol{u}_{f}=0 \quad \text { in } \Omega_{f} \\
(1)
\end{gathered}
$$

where $\boldsymbol{u}_{\boldsymbol{f}}$ and $p_{f}$ are the unknown fluid velocity and pressure, respectively, while $\boldsymbol{g}$ is gravitational acceleration and

$$
T\left(\mu ; \boldsymbol{u}_{f}, p_{f}\right)=-p_{f} \boldsymbol{I}+\mu\left(\nabla \boldsymbol{u}_{f}+\left(\nabla \boldsymbol{u}_{f}\right)^{\mathrm{T}}\right)
$$


is the Cauchy stress tensor. For this equation to be a valid model for our system, we have to determine the operating velocity regime (laminar or turbulent); the common practice is to evaluate the Reynolds number [67]:

$R e_{f}=\frac{\rho U_{f} L_{f}}{\mu}$

$R e_{f}$ is sufficiently low, a valid simplification of the Navier-Stokes equations (1) is the Stokes equation that can be obtained by neglecting the inertia term $\rho\left(\boldsymbol{u}_{\boldsymbol{f}} \cdot \nabla\right) \boldsymbol{u}_{\boldsymbol{f}}$. This makes the momentum equation linear and thus much easier to numerically solve, but this is only valid for very low velocities, densities and characteristic lengths or highly viscous fluids. The Navier-Stokes equations (1) are generally considered valid for $R e_{f}<$ 2000 [68]. For Reynolds numbers above 2000, the flow is said to be affected by turbulence and another model must be introduced, with the most commonly used in CFD being the $k$ - $\varepsilon$ model [69] or the Reynolds averaged Navier-Stokes (RANS) model [70]. It is not in the scope of this paper to review those cases, as in most MF and UF membrane-based systems, the velocity magnitude is usually low so that $R e_{f}$ does not exceed the turbulence threshold. Recent examples of CFD studies on tubular membranes that include turbulence are found in $[71,25,72]$ where baffles and spacers are used to obstruct flow in the geometry in order to achieve better filtration performance by reducing fouling and increasing the flux.

Darcy's law is a linear relation between velocity $\boldsymbol{u}_{\boldsymbol{p}}$ and the pressure $p_{p}$ of an incompressible fluid in a saturated porous medium [73]:

$$
\begin{array}{r}
\boldsymbol{u}_{\boldsymbol{p}}=-\frac{\boldsymbol{K}}{\boldsymbol{\mu}}\left(\nabla p_{p}-\rho \boldsymbol{g}\right) \text { in } \Omega_{p} \\
\nabla \cdot \boldsymbol{u}_{\boldsymbol{p}}=0 \text { in } \Omega_{p}
\end{array}
$$

with $\boldsymbol{K}$ the permeability tensor. If the porous medium can be assumed homogeneous and isotropic as it is often the case for membranes, $\boldsymbol{K}$ can be replaced by a constant $\mathrm{K}$. Typical values of $\mathrm{K}$ for UF membranes are in the range $10^{-15} \leq K \leq 10^{-9} \mathrm{~m}^{2}$ (see, e.g., $[12,74])$.

Darcy's law is considered valid for porous Reynolds number (considering the characteristic length as the average pore radius) below 10 (see, e.g., [75]). The porosity of the membrane is also a deciding factor for the equations to be used: $[63,76]$ report that a threshold of 0.6 can be used for the porosity, above which the shear stresses inside the porous medium are considered to become non-negligible. In such cases, non-Darcian models are used as discussed, e.g., [77]. One of these models is Brinkman equation [78] that adapts the Navier-Stokes momentum equations by adding the Darcian term $\mu K^{-1} \boldsymbol{u}_{\boldsymbol{p}}$ to mimic the presence of a porous medium. The problem translates into finding the velocity $\boldsymbol{u}_{\boldsymbol{p}}$ and the pressure $p_{p}$ such that

$$
\begin{gathered}
\rho\left(\boldsymbol{u}_{\boldsymbol{p}} \cdot \nabla\right) \boldsymbol{u}_{\boldsymbol{p}}=\nabla \cdot T\left(\mu_{b} ; \boldsymbol{u}_{\boldsymbol{p}}, p_{p}\right)- \\
\mu K^{-1} \boldsymbol{u}_{\boldsymbol{p}}+\rho \boldsymbol{g} \text { in } \Omega_{p} \\
\nabla \cdot \boldsymbol{u}_{\boldsymbol{p}}=0 \text { in } \Omega_{p}
\end{gathered}
$$

Equation (4) $)_{1}$ involves the effective (or Brinkman) viscosity $\mu_{b}$ instead of $\mu$, which should be estimated experimentally since there are no theoretical expressions for it [79]. For instance, in [80], experimental work on idealized axial flow through infinite and streaked arrays of cylindrical rods showed a dependence of the effective viscosity on porosity and that $\mu_{b} \leq \mu$ for their particular case. Furthermore, [81] relates $\mu_{b}$ to the slip coefficient on the interface between a free fluid and a porous medium. However, due to a lack of general consensus on how to 
determine $\mu_{b}$, it is generally accepted to approximate $\mu_{b} \approx \mu$ [82].

Another non-Darcian model is Forchheimer equation [83, 84, 85]. In this case, a quadratic non-linear term is added to Darcy's law to account for the viscous dissipation (conversion of kinetic energy into internal energy by work done against the viscous stress) as mentioned in [86]. The Forchheimer equations become

$$
\begin{gathered}
-\nabla p_{p}=-\frac{\mu}{K} \boldsymbol{u}_{\boldsymbol{p}}+\frac{\rho C_{f}}{\sqrt{K}}\left|\boldsymbol{u}_{\boldsymbol{p}}\right| \boldsymbol{u}_{\boldsymbol{p}}+\rho \boldsymbol{g} \text { in } \Omega_{p} \\
\nabla \cdot \boldsymbol{u}_{\boldsymbol{p}}=0 \text { in } \Omega_{p}
\end{gathered}
$$

where $\boldsymbol{u}_{\boldsymbol{p}}$ and $p_{p}$ are the velocity and pressure, respectively, inside the porous medium and $C_{f}$ is the Forchheimer drag (or inertial resistance) coefficient $[85,86]$. (In general, nonlinear correction terms of the form $\left|\boldsymbol{u}_{\boldsymbol{p}}\right|^{\alpha} \boldsymbol{u}_{\boldsymbol{p}} \quad$ with $1 \leq \alpha \leq 2$ can be considered for Darcy's law as discussed in [87].)

Considering the Navier-Stokes equations (1) in $\Omega_{f}$ and Darcy's law (3) or the Brinkman equation (4) or Forchheimer equation (5) in $\Omega_{p}$, we have to introduce coupling conditions prescribed on the common free flowporous interface $\Gamma_{I}$, in order to accurately represent the filtration process.

A classical set of conditions introduced to couple the Navier-Stokes equations and Darcy's law as seen, e.g., in $[52,88,89,90,91,92]$ are:

i) the continuity of the normal velocity across $\Gamma_{I}$ :

$\boldsymbol{u}_{\boldsymbol{f}} \cdot \mathbf{n}=\boldsymbol{u}_{\boldsymbol{p}} \cdot \mathbf{n}$ on $\Gamma_{I}$

ii) the balance of the normal stresses across $\Gamma_{I}$

$p_{p}+\rho g z=-\mathbf{n} \cdot T\left(\mu ; \boldsymbol{u}_{\boldsymbol{f}}, p_{f}\right) \cdot \mathbf{n}$ on $\Gamma_{I}$

where $z$ is the elevation with respect to a reference level, and

iii) the Beavers-Joseph-Saffman condition on the Navier-Stokes tangential velocity component:

$\mathbf{t} \cdot T\left(\mu ; \boldsymbol{u}_{f}, p_{f}\right) \cdot \mathbf{n}=-\mathbf{t} \cdot\left(\frac{\alpha \mu}{\sqrt{K}} \boldsymbol{u}_{f}\right)$ on $\Gamma_{I}$

Here, $\mathbf{n}$ and $\mathbf{t}$ are the normal (pointing outwards of $\Omega_{f}$ ) and tangential unit vectors on $\Gamma_{I}$, while $\alpha$ is the dimensionless Beavers-JosephSaffman slip coefficient that depends on the characteristics of the porous medium [52]. According to the classical experiments of [52], a valid range for the slip coefficient for different porous materials is $0.1 \leq \alpha \leq$ 4.

The coupling conditions indicated in equations (6)-(8) apply also in the case Forchheimer equations (5) are used in the porous medium domain $\Omega_{p}$.

To couple the Navier-Stokes and Brinkman equations, a possible strategy is to impose the continuity of both velocity and normal stress [81, 93]:

$\boldsymbol{u}_{\boldsymbol{p}}=\boldsymbol{u}_{\boldsymbol{f}}$ on $\Gamma_{I}$

$T\left(\mu_{b} ; \boldsymbol{u}_{\boldsymbol{p}}, p_{p}\right) \cdot \mathbf{n}=T\left(\mu ; \boldsymbol{u}_{f}, p_{f}\right) \cdot \mathbf{n}$ on $\Gamma_{I}$

whereas other authors suggest replacing equation $(9)_{2}$ by the following jump condition for the tangential component of the stress [94]:

$$
\left(\varepsilon_{p}^{-1} \nabla \boldsymbol{u}_{p}-\nabla \boldsymbol{u}_{f}\right) \cdot \mathbf{n}=\frac{\beta}{\sqrt{K}} \boldsymbol{u}_{\boldsymbol{p}}
$$

where $\varepsilon_{p}$ is the porosity (liquid volume fraction) of the porous medium and $\beta$ is a dimensionless coefficient to be determined experimentally.

One-domain Approach. The difficulty of identifying appropriate interface coupling conditions and of solving different types of equations in 
two sub-regions of the domain of interest is avoided by using the onedomain approach [66].

This method is commonly found in commercial finite element software (such as, e.g., COMSOL Multiphysics [95]), since it is much more straightforward to implement. It uses spatially changing coefficients (porosity and permeability) within a unified momentum equation, to realistically model filtration. More precisely, the problem proposed in [66] requires to compute the fluid velocity $\boldsymbol{u}$ and pressure $p$ in the whole domain $\Omega$ such that

$\rho\left(\frac{\boldsymbol{u}}{\varepsilon_{p}} \cdot \nabla\right) \frac{\boldsymbol{u}}{\varepsilon_{p}}=\nabla \cdot T\left(\mu_{b} \varepsilon_{p}^{-1} ; \boldsymbol{u}, p\right)-$

$\mu \widetilde{K} \boldsymbol{u}+\rho \boldsymbol{g}$ in $\Omega$

$\nabla \cdot \boldsymbol{u}=0$ in $\Omega$

where the porosity $\varepsilon_{p}$ is set equal to 1 in $\Omega_{f}$, while $\widetilde{K}$ is zero in $\Omega_{f}$. Naturally, $\widetilde{K}=K^{-1}$ in $\Omega_{p}$ and $\varepsilon_{p}$ assumes the value of the porous medium porosity in $\Omega_{p}$. Using this model, both velocity and pressure are continuous across the interface $\Gamma_{I}$ but an interfacial stress jump is induced due to the discontinuous porosity and the Darcian term $\mu \widetilde{K} \boldsymbol{u}$ that plays the role of an additional stress term in the porous medium domain. A comparison between the coupled Stokes/Darcy model and this approach is carried out in [66]. The finite element approximation of the one-domain

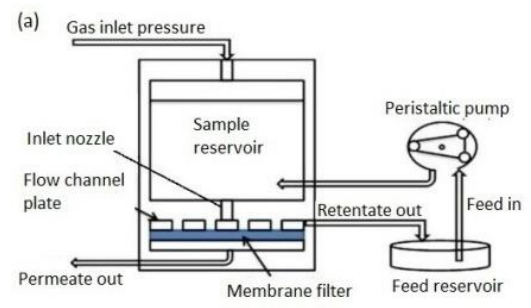

model has been studied extensively in [96].

An extension of the one-domain equations (10) to account for the case of non-Darcian flow regimes in the porous medium is provided by the socalled penalization method [97, 63]. This is analogous to the one-domain approach, since it consists of a modified set of Navier-Stokes equations in the whole domain of interest, including two penalization terms associated to the resistance induced by the porous medium. More precisely, this model looks for the fluid velocity $\boldsymbol{u}$ and pressure $p$ such that

$\rho\left(\frac{\boldsymbol{u}}{\varepsilon_{p}} \cdot \nabla\right) \frac{\boldsymbol{u}}{\varepsilon_{p}}=\nabla \cdot T\left(\mu_{b} \varepsilon_{p}^{-1} ; \boldsymbol{u}, p\right)-$

$\mu \widetilde{K} \boldsymbol{u}-\rho C_{f} \sqrt{\widetilde{K}}|\boldsymbol{u}| \boldsymbol{u}+\rho \boldsymbol{g}$ in $\Omega$

$\nabla \cdot \boldsymbol{u}=0$ in $\Omega$.

This model does not require coupling conditions and it is used in commercial CFD software (e.g., $[98,95])$.

All the models presented produce analogous results both in the fluid region and inside the porous medium. The most notable differences are in the interfacial region where the onedomain and the penalization models predict a continuous velocity field while the other three models result in a discontinuous tangential velocity. A comparison between the different approaches can be found in [63] for a simple 2D setting where these differences can be easily appreciated.

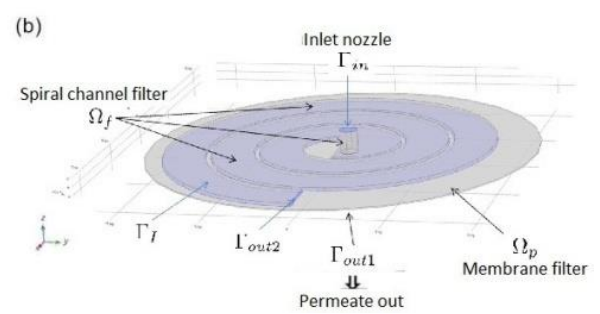

Figure 3 Schematic representation of the filtration device (left) and 3D illustration of the spiral flow channel and the membrane filter 


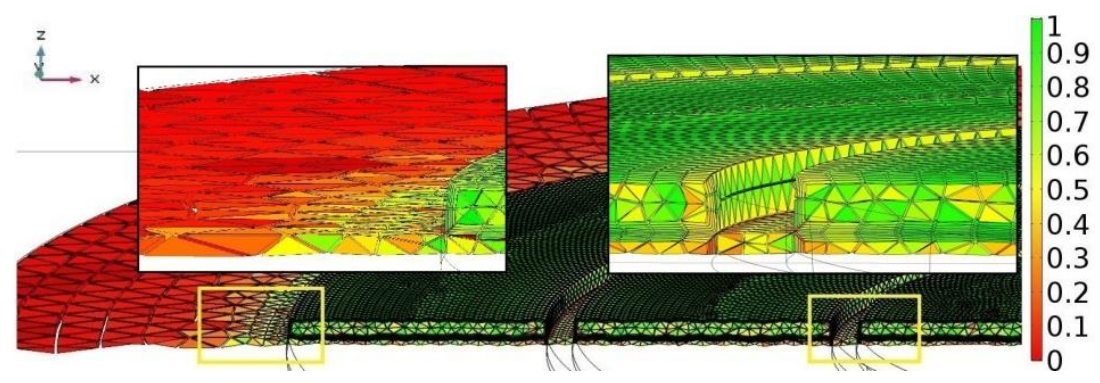

Figure 4 Mesh created in COMSOL made up of 659,139 elements

\subsection{SOLVING USING SOFTWARE: COMSOL}

In this section, we give an example of how the Navier-Stokes/Darcy and the one-domain models can be solved using COMSOL Multiphysics 5.3a [95], a commercial finite element solver that can consider multi-physics problems characterized by different equations in different regions as well as various types of boundary and coupling conditions.

Consider the hybrid water purification system illustrated in Figure 3. The fluid flows from the sample reservoir into a spiral-shaped channel with open bottom placed on top of a
UF membrane sheet $[99,100,101]$. We remark that the computational domain is characterized by different characteristic lengths since the membrane is much thinner than the free flow domain (the spiral channel), a common situation also for other applications (see, e.g., [38]). Thus, anisotropic meshes made of tetrahedra and prisms refined both inside the free flow channel and in the membrane should be considered. An example of computational mesh generated using COMSOL is shown in Figure 4 where we can see that boundary layers were added near the interface and on the channel walls, while coarser elements were used inside the membrane where small velocity gradients are expected.
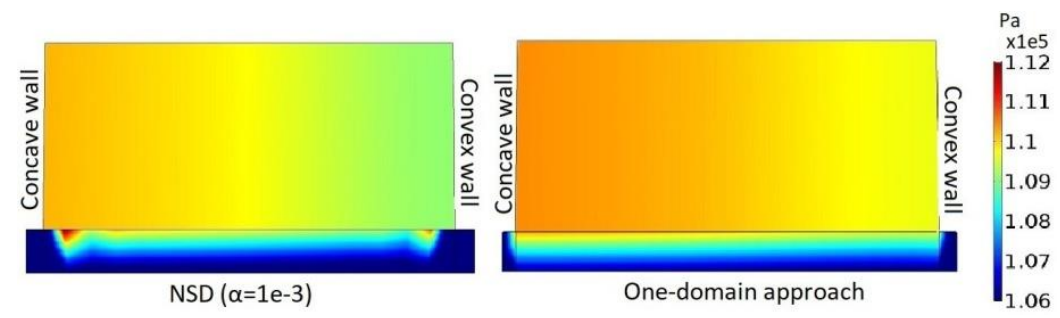

Figure 5 Pressure computed using the Navier-Stokes/Darcy model (left) and the one-domain model (right) at a cross-section of the device

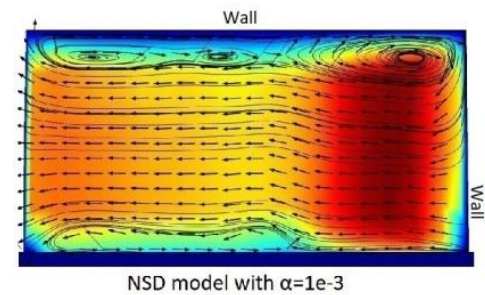

NSD model with $\alpha=1 \mathrm{e}-3$

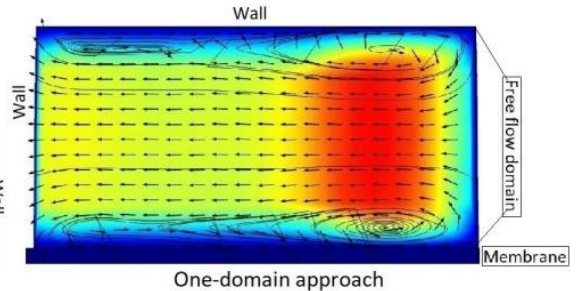

One-domain approach

Figure 6 Velocity magnitude at a cross section of the device using Navier- Stokes/Darcy (left) and the one-domain model (right). Dean vortices are visible for both models 


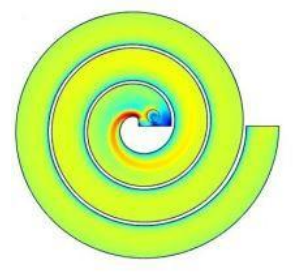

NSD $\alpha=1 \mathrm{e}-3$

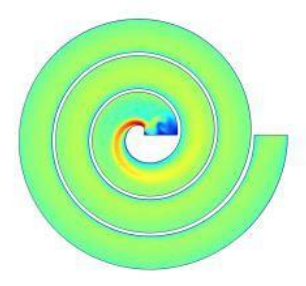

NSD $\alpha=1$

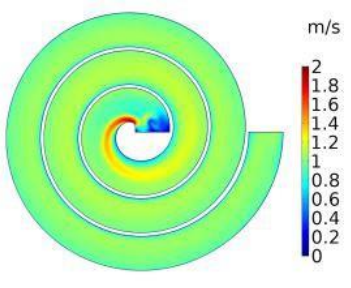

One-domain

Figure 7 Velocity magnitude plotted in the middle of the spiral channel for the two models

The finite element approximation of the Navier-Stokes and the one-domain momentum equations can be realized by Lagrangian elements. COMSOL uses the second-order Taylor-Hood $P_{2}-P_{1}$ elements by default but $P_{1}-$ $P_{1}$ elements with streamline stabilization [102, 103, 104] can be easily selected to reduce the computational cost by reducing the number of unknowns for the velocity field. In order to solve Darcy's law (3), COMSOL automatically reduces the system of equations to an elliptic equation for the pressure: find $p_{p}$ such that

$$
\nabla \cdot\left(-\frac{K}{\mu} \nabla p_{p}\right)=0 \text { in } \Omega_{p}
$$

and then it uses Darcy's law in equation (3) 1 to post-process the velocity. To discretize the pressure $p_{p}$, one can use $P_{1}$ Lagrangian elements, which is the simplest and most economical choice in terms of computational cost. Since COMSOL solves this elliptic equation with the pressure as the only unknown, instead of solving the mixed formulation (3), the system that arises from the finite element approximation of the NavierStokes/Darcy model has fewer unknowns (degrees of freedom) than the one associated with the one-domain model. In particular, the mesh in Figure 4 has 659,139 elements that correspond to 829,852 unknowns (for velocity and pressure) for the Navier-Stokes/Darcy problem and to $1,080,036$ unknowns for the one-domain case.

Suitable boundary conditions must be introduced to represent the presence of the inlets, outlets and the impermeable walls. Additionally, the coupling conditions considered in Sect. 3.3.1 for the coupled heterogeneous problems can be implemented in COMSOL as if they were boundary conditions for each sub-problem in the respective sub-domain, either $\Omega_{f}$ or $\Omega_{p}$. More precisely, the BeaversJoseph-Saffman condition (equation (8)) and the continuity of normal stresses (equation (7)) should be implemented as boundary stresses for the Navier-Stokes equations (1). For Darcy's law (equation (12)) the continuity of normal velocity, equation (6), should be imposed as a Neumann boundary condition for the pressure.

The finite element approximations give rise to non-linear systems of equations that were solved using Newton's method with convergence criterion requiring that the residual error must be less than $10^{-3}$.

In Figure 5, we plot the computed pressure on a cross section of the channel for a given inflow pressure. We remark that, while the one-domain model gives a continuous pressure across the interface, a pressure jump arises in the Navier-Stokes/Darcy case due to the imposed coupling conditions. Moreover, the centrifugal force produced by the curved shape of the fluid channels creates a pressure gradient between the convex and the 
concave walls. As expected, this gives rise to Dean vortices that are clearly visible in Figure 6. Velocity vectors and streamlines highlight the vortices near the membrane surface and at the top of the channel as discussed in [100, 101].

Finally, Figure 7 shows the velocity magnitude plotted in the middle of the spiral channel for the one-domain model and for the Navier-Stokes/Darcy model for different values of the BeaversJoseph-Saffman slip coefficient $\alpha$. The lowest value of $\alpha$ corresponds to higher slip velocity on the interface as clearly shown in the figure.

\subsection{CONCLUSIONS}

In this paper, we have reviewed several modeling techniques that have been proposed in the literature to represent filtration in membrane systems for water treatment applications. It is evident that many models rely on strong assumptions on the flow behavior and are application specific so that they cannot be applied to general configurations, such as hybrid membrane filtration systems. Additionally, we have identified the basic mesoscopic models that can be used to model the fluid dynamics in generic configurations. We have also demonstrated how the proposed settings could be implemented in a commercial finite element software to simulate filtration inside a specific water-purification device. Overall, we conclude that while the literature on coupled free and porous modelling is mature, there is still scope to apply these approaches to modeling filtration systems, due to the dynamic nature of these domain, and this is something that should be pursued vigorously in the future.

\section{REFERENCES}

[1] N. Shamsuddin, D. B. Das, and V. M. Starov. 2016. Membranebased Point-of-use Water Treatment (PoUWT) System in Emergency Situations. Sep. Purif. Rev. 45(1): 50-67.

[2] D. Lantagne and T. Clasen. 2013. Effective Use of Household Water Treatment and Safe Storage in Response to the 2010 Haiti Earthquake. Am. J. Trop. Med. Hyg. 89(3): 426-433.

[3] B. Michen, F. Meder, A. Rust, J. Fritsch, C. Aneziris, and T. Graule. 2012. Virus Removal in Ceramic Depth Filters Based on Diatomaceous Earth. Environ. Sci. Technol. 46(2): 1170-1177.

[4] B. A. Ferrier and J. T. Spickett. 2007. Natural Disasters in Developing Countries: An Environmental Health Perspective. Asia-Pac. J. Public Health. 19(1 suppl): 18-24.

[5] N. Shamsuddin, C. Cao, V. M. Starov, and D. B. Das. 2016. A Comparative Study between Stirred Dead End and Circular Flow in Microfiltration of China Clay Suspensions. Water Sci. Technol.- Water Supply. 16: 481492.

[6] S. Metsämuuronen, M. Sillanpää, A. Bhatnagar, and M. Mänttäri. 2014. Natural Organic Matter Removal from Drinking Water by Membrane Technology. Sep. Purif. Rev. 43(1): 1-61.

[7] M. Peter-Varbanets, C. Zurbrügg, C. Swartz, and W. Pronk. 2009. Decentralized Systems for Potable Water and the Potential of Membrane Technology. Water Res. 43(2): 245-265.

[8] R. Sondhi, R. Bhave, and G. Jung. 2003. Applications and Benefits of Ceramic Membranes. 
Membrane Technology. 2003(11): 5-8.

[9] R. W. Baker. 2012. Membrane Technology and Applications. Wiley.

[10] E. S. Tarleton and R. J. Wakeman. 2008. Dictionary of Filtration and Separation. Filtration Solutions.

[11] R. Wakeman and S. Tarleton. 2005. Solid/Liquid Separation: Principles of Industrial Filtration. Elsevier Science.

[12] J. Mulder. 1996. Basic Principles of Membrane Technology. Springer.

[13] P. Bacchin, D. Si-Hassen, V. Starov, M. J. Clifton, and P. Aimar. 2002. A Unifying Model for Concentration Polarization, Gel-layer Formation and Particle Deposition in Crossflow Membrane Filtration of Colloidal Suspensions. Chem. Eng. Sci. 57(1): 77-91.

[14] J. Mendret, C. Guigui, P. Schmitz, and C. Cabassud. 2009. In Situ Dynamic Characterisation of Fouling Under Different Pressure Conditions during Dead-end Filtration: Compressibility Properties Of Particle Cakes. J. Membr. Sci. 333(1): 20-29.

[15] B. Blankert, B. Betlem, and B. Roffel. 2006. Dynamic Optimization of a Dead-end Filtration Trajectory: Blocking Filtration Laws. J. Membr. Sci. 285(1): 90-95.

[16] A. Gugliuzza. 2015. Membrane Wettability. In E. Drioli and L. Giorno, (Editor). Encyclopedia of Membranes. Springer.

[17] C. E. Goodyer and A. L. Bunge. 2012. Mass Transfer through Membranes with Surface Roughness. J. Membr. Sci. 409410: 127-136.
[18] H. Suhaimi, S. Wang, T. Thornton, and D. B. Das. 2015. On Glucose Diffusivity of Tissue Engineering Membranes and Scaffolds. Chem. Eng. Sci. 126: 244-256.

[19] W. R. Dean. 1928. Fluid Motion in a Curved Channel. Proc. $R$. Soc. London Ser. A-Math. Phys. Eng. Sci. 121(787): 402-420.

[20] N. Nivedita, P. Ligrani, and Papautsky. 2017. Dean Flow Dynamics in Low-aspect Ratio Spiral Microchannels. Sci. Rep. 7(44072).

[21] G. Belfort. 1989. Fluid Mechanics in Membrane Filtration: Recent Developments. J. Membr. Sci. 40(2): 123-147.

[22] B. Olayiwola and P. Walzel. 2009. Effects of In-phase Oscillation of Retentate and Filtrate in Crossflow Filtration at Low Reynolds Number. $J$. Membr. Sci. 345(1): 36-46.

[23] P. Wei, K. Zhang, W. Gao, L. Kong, and R. Field. 2013. CFD Modeling of Hydrodynamic Characteristics of Slug Bubble Flow in a Flat Sheet Membrane Bioreactor. J. Membr. Sci. 445: 15-24.

[24] T. Taha and Z. F. Cui. 2002. CFD Modelling of Gas-sparged Ultrafiltration in Tubular Membranes. J. Membr. Sci. 210(1): 13-27.

[25] S. Ahmed, M. Seraji, J. Jahedi, and M. A. Hashib. 2012. Application of CFD for Simulation of a Baffled Tubular Membrane. Chem. Eng. Res. Des. 90(5): 600-608.

[26] H. S. Abid, D. J. Johnson, R. Hashaikeh, and N. Hilal. 2017. A Review of Efforts to Reduce Membrane Fouling by Control of Feed Spacer Characteristics. Desalination. 420: 384-402. 
[27] M. Shakaib, S. M. F. Hasani, and M. Mahmood. 2007. Study on the Effects of Spacer Geometry in Membrane Feed Channels using Three-dimensional Computational Flow Modeling. $J$. Membr. Sci. 297(1): 74-89.

[28] C. P. Koutsou, S. G. Yiantsios, and A. J. Karabelas. 2007. Direct Numerical Simulation of Flow in Spacer-filled Channels: Effect of Spacer Geometrical Characteristics. J. Membr. Sci. 291(1): 53-69.

[29] C. P. Koutsou and A. J. Karabelas. 2015. A Novel Retentate Spacer Geometry for Improved Spiral Wound Membrane (SWM) Module Performance. J. Membr. Sci. 488: 129-142.

[30] K. K. Lau, M. Z. Abu Bakar, L. Ahmad, and T. Murugesan. 2009. Feed Spacer Mesh Angle: 3D Modeling, Simulation and Optimization based on Unsteady Hydrodynamic in Spiral Wound Membrane Channel. J. Membr. Sci. 343(1): 16-33.

[31] G. A. Fimbres-Weihs and D. E. Wiley. 2010. Review of 3D CFD Modeling of Flow and Mass Transfer in Narrow Spacer-filled Channels in Membrane Modules. Chem. Eng. Process. 49(7): 759781.

[32] G. Belfort and N. Nagata. 1985. Fluid Mechanics and Crossflow Filtration: Some Thoughts. Desalination. 53(1): 57-79.

[33] P. Schmitz, D. Houi, and A. Wandelt. 1992. Hydrodynamic Aspects of Crossflow Microfiltration. Analysis of Particle Deposition at the Membrane Surface. J. Membr. Sci. 71(1): 29-40.

[34] B. Gu, C. S. Adjiman, and X. Y. Xu. 2017. The Effect of Feed Spacer Geometry on Membrane
Performance and Concentration Polarisation based on 3D CFD Simulations. J. Membr. Sci. 527: 78-91.

[35] A. L. Ahmad, K. K. Lau, and M. Z. Abu Bakar. 2005. Impact of Different Spacer Filament Geometries on Concentration Polarization Control in Narrow Membrane Channel. J. Membr. Sci. 262: 138-152.

[36] A. S. Berman. 1953. Laminar Flow in Channels with Porous Walls. J. Appl. Phys. 24(9): 1232-1235.

[37] C. Kleinstreuer and M. S. Paller. 1983. Laminar Dilute Suspension Flows in Plate-and-frame Ultrafiltration Units. AICHE $J$. 29(4): 529-533.

[38] L. Huang and M. T. Morrissey. 1999. Finite Element Analysis as a Tool for Crossflow Membrane Filter Simulation. J. Membr. Sci. 155(1): 19-30.

[39] V. Nassehi. 1998. Modelling of Combined Navier-stokes and Darcy Flows in Crossflow Membrane Filtration. Chem. Eng. Sci. 53(6): 1253-1265.

[40] C. J. Richardson and V. Nassehi. 2003. Finite Element Modelling of Concentration Profiles in Flow Domains with Curved Porous Boundaries. Chem. Eng. Sci. 58(12): 2491-2503.

[41] S. Wardeh and H. P. Morvan. 2008. CFD Simulations of Flow and Concentration Polarization in Spacer-filled Channels for Application to Water Desalination. Chem. Eng. Res. Des. 86(10): 1107-1116.

[42] D. F. Fletcher and D. E. Wiley. 2004. A Computational Fluids Dynamics Study of Buoyancy Effects in Reverse Osmosis. $J$. Membr. Sci. 245(1): 175-181.

[43] P. Schmitz and M. Prat. 1995. 3D Laminar Stationary Flow Over 
a Porous Surface with Suction: Description at Pore Level. AICHE J. 41(10): 2212-2226.

[44] E. Lyster and Y. Cohen. 2007. Numerical Study of Concentration Polarization in a Rectangular Reverse Osmosis Membrane Channel: Permeate Flux Variation and Hydrodynamic End Effects. $J$. Membr. Sci. 303(1): 140-153.

[45] P. Schausberger, N. Norazman, H. Li, V. Chen, and A. Friedl. 2009. Simulation of Protein Ultrafiltration using CFD: Comparison of Concentration Polarisation and Fouling Effects with Filtration and Protein Adsorption Experiments. $J$. Membr. Sci. 337(1): 1-8.

[46] K. Damak, A. Ayadi, B. Zeghmati, and P. Schmitz. 2004. A New Navier-Stokes and Darcy's Law Combined Model for Fluid Flow in Crossflow Filtration Tubular Membranes. Desalination. 161(1): 67-77.

[47] Y. M. J. Chew, W. R. Paterson, and D. I. Wilson. 2007. Fluid Dynamic Gauging: A New Tool to Study De- Position on Porous Surfaces. J. Membr. Sci. 296(1): 29-41.

[48] V. Y. Lister, C. Lucas, P. W. Gordon, Y. M. Chew, and D. I. Wilson. 2011. Pressure Mode Fluid Dynamic Gauging for Studying Cake Build-up in Crossflow Microfiltration. $J$. Membr. Sci. 366(1): 304-313.

[49] V. Geraldes, V. Semião, and M. Norberta de Pinho. 2001. Flow and Mass Transfer Modelling of Nanofiltration. J. Membr. Sci. 191(1): 109-128,

[50] M. A. Monfared, N. Kasiri, A. Salahi, and T. Mohammadi. 2012. Modeling Ultrafiltration of Gelatin-water Suspension by Computational Fluid Dynamics.
Chem. Eng. Res. Des. 90(8): 1098-1104.

[51] H. M. Yeh and T. W. Cheng. 1999. Analysis of the Slip Effect on the Permeate Flux in Membrane Ultrafiltration. $J$. Membr. Sci. 154(1): 41-51,

[52] G. S. Beavers and D. D. Joseph. 1967. Boundary Conditions at a Naturally Permeable Wall. $J$. Fluid Mech. 30: 197-207.

[53] M. F. Gruber, C. J. Johnson, C. Y. Tang, M. H. Jensen, L. Yde, and C. Hélix-Nielsen. 2011. Computational Fluid Dynamics Simulations of Flow and Concentration Polarization in Forward Osmosis Membrane Systems. J. Membr. Sci. 379(1): 488-495.

[54] E. V. Mosina. 2010. Numerical Study of Flow at a Liquid-porous Medium Interface. Theor. Found. Chem. Eng. 44(5): 679-685.

[55] M. Kostoglou and A. J. Karabelas. 2009. On the Fluid Mechanics of Spiral-wound Membrane Modules. Ind. Eng. Chem. Res. 48(22): 10025-10036.

[56] Z. Jalilvand, F. Z. Ashtiani, A. Fouladitajar, and H. Rezaei. 2014. Computational Fluid Dynamics Modeling and Experimental Study of Continuous and Pulsatile Flow In Flat Sheet Microfiltration Membranes. J. Membr. Sci. 450: 207-214.

[57] A. J. Karabelas, M. Kostoglou, and C. P. Koutsou. 2015. Modeling of Spiral Wound Membrane Desalination Modules and Plants - Review and Research Priorities. Desalination. 356: 165-186.

[58] H. Lotfiyan, F. Z. Ashtiani, A. Fouladitajar, and S. B. Armand. 2014. Computational Fluid Dynamics Modeling and Experimental Studies of Oil-in- 
Water Emulsion Microfiltration in a Flat Sheet Membrane Using Eulerian Approach. J. Membr. Sci. 472: 1-9.

[59] G. Keir and V. Jegatheesan. 2014. A Review of Computational Fluid Dynamics Applications in Pressure-driven Membrane Filtration. Rev. Environ. Sci. Bio-Technol. 13(2): 183-201.

[60] V. V. Tarabara and M. R. Wiesner. 2003. Computational Fluid Dynamics Modeling of the Flow in a Laboratory Membrane Filtration Cell Operated at Low Recoveries. Chem. Eng. Sci. 58(1): 239-246.

[61] Z. Yang, J. Cheng, C. Yang, and B. Liang. 2016. CFD-based Optimization and Design of Multi-channel Inorganic Membrane Tubes. Chin. J. Chem. Eng. 24(10): 1375-1385.

[62] S. Chellam, M. R. Wiesner, and C. Dawson. 1992. Slip at a Uniformly Porous Boundary: Effect on Fluid Flow and Mass Transfer. J. Eng. Math. 26(4): 481-492.

[63] F. Cimolin and M. Discacciati. 2013. NavierStokes/Forchheimer Models for Filtration through Porous Media. Appl. Numer. Math. 72: 205-224.

[64] N. S. Hanspal, A. N. Waghode, V. Nassehi, and R. J. Wakeman. 2006. Numerical Analysis of Coupled Stokes/Darcy Flows in Industrial Filtrations. Transp. Porous Media. 64(1): 73.

[65] N. S. Hanspal, A. N. Waghode, V. Nassehi, and R. J. Wakeman. 2009. Development of a Predictive Mathematical Model for Coupled Stokes/Darcy Flows in Crossflow Membrane Filtration. Chem. Eng. J. 149(1): 132-142.
[66] M. Le Bars and M. Grae Worster. 2006. Interfacial Conditions between a Pure Fluid and a Porous Medium: Implications for Binary Alloy Solidification. $J$. Fluid Mech. 550: 149-173.

[67] O. Reynolds. 1883. An Experimental Investigation of the Circum- Stances which Determine Whether the Motion of Water Shall Be Direct or Sinuous, and of the Law of Resistance in Parallel Channel. Phil. Trans. R. Soc. 174: 935-982.

[68] M. J. Rhodes. 1998. Introduction to Particle Technology. Wiley.

[69] K. Hanjalić and B. E. Launder. 1972. A Reynolds Stress Model of Turbulence and Its Application to Thin Shear Flows. J. Fluid Mech. 52(4): 609-638.

[70] D. C. Wilcox. 2008. Formulation of the $\mathrm{k}-\omega$ Turbulence Model Revisited. AIAA J. 52.

[71] S. Ahmed, M. Seraji, J. Jahedi, and M. A. Hashib. 2011. CFD Simulation of Turbulence Promoters in a Tubular Membrane Channel. Desalination. 276(1): 191-198.

[72] M. Jafarkhani, M. K. Moraveji, R. Davarnejad, F. Moztarzadeh, and M. Mozafari. 2012. Threedimensional Simulation of Turbulent Flow in a Membrane Tube Filled with Semi-circular Baffles. Desalination. 294: 8-16.

[73] H. Darcy. 1856. Les Fontaines Publiques de la Ville de Dijon. Dalmont, Paris.

[74] K. Scott. 1996. Handbook of Industrial Membranes. Elsevier Science.

[75] K. R. Arora. 2009. Soil Mechanics and Foundation Engineering. Standard Publishing.

[76] V. Nassehi and D. B. Das. 2007. Computational Methods in the Management of Hydro- 
environmental Systems. IWA Publishing.

[77] Z. Zeng and R. Grigg. 2006. A Criterion for Non-Darcy Flow in Porous Media. Transp. Porous Media. 63(1): 57-69.

[78] H. C. Brinkman. 1947. A Calculation of the Viscous Force Exerted by a Flowing Fluid on a Dense Swarm of Particles. Appl. Sci. Res. A. 1: 27-34.

[79] L. Durlofsky and J. F. Brady. 1987. Analysis of the Brinkman Equation as a Model for Flow in Porous Media. Phys. Fluids. 30(11): 3329-3341.

[80] J. A. Kolodziej. 1988. Influence of the Porosity of a Porous Medium on the Effective Viscosity in Brinkman's Filtration Equation. Acta Mech. 75(1): 241-254.

[81] G. Neale and W. Nader. 1974. Practical Significance of Brinkman's Extension of Darcy's Law: Coupled Parallel Flows within a Channel and a Bounding Porous Medium. Can. J. Chem. Eng. 52(4): 475-478.

[82] K. Vafai and R. Thiyagaraja. 2002. Analysis of Flow and Heat Transfer at the Interface Region of a Porous Medium. Int. J. Heat Mass Transf. 30: 1391-1405.

[83] P. Forchheimer. 1901. Wasserbewegung durch Boden. Zeitschrift des Vereines Deutscher Ingenieuer. 45: 17821788.

[84] S. Whitaker. 1999. The Method of Volume Averaging. Springer.

[85] V. A. Jambhekar. 2011. Forchheimer Porous-media Flow Models. Numerical Investigation and Comparison with Experimental Data. Master's Thesis. Universität Stuttgart.

[86] D. Takhanov. 2011. Forchheimer Model for Non-Darcy Flow in Porous Media and Fractures.
Master's Thesis. Imperial College London.

[87] M. Firdaouss, J. L. Guermond, and P. Le Quere. 1997. Nonlinear Corrections to Darcy's Law at Low Reynolds Numbers. J. Fluid Mech. 343: 331-350.

[88] P.G. Saffman. 1971. On the Boundary Condition at the Interface of a Porous Medium. Stud. Appl. Math. 1: 93-101.

[89] W. Jäger and A. Mikelić. 1996. On the Boundary Conditions at the Contact Interface between a Porous Medium and a Free Fluid. Ann. Scuola Norm. Sup. Pisa Cl. Sci. 23: 403-465.

[90] M. Discacciati, E. Miglio, and A. Quarteroni. 2002. Mathematical and Numerical Models for Coupling Surface and Groundwater Flows. Appl. Numer. Math. 43: 57-74.

[91] W. L. Layton, F. Schieweck, and I. Yotov. 2003. Coupling Fluid Flow with Porous Media Flow. SIAM J. Numer. Anal. 40: 21952218.

[92] V. Girault and B. Rivière. 2009. DG Approximation of Coupled Navier-Stokes and Darcy equations by Beaver-JosephSaffman Interface Condition. SIAM J. Numer. Anal. 47: 20522089.

[93] O. Iliev and V. Laptev. 2004. On Numerical Simulation of Flow through Oil Filters. Comput. Vis. Sci. 6(2): 139-146.

[94] A. Ochoa Tapia and S. Whitaker. 1995. Momentum Transfer at the Boundary between a Porous Medium and a Homogeneous Fluid I. Theoretical Development. Int. J. Heat Mass Transf. 38: 2635-2646.

[95] COMSOL AB. Comsol Multiphysics version 5.3a. 2017. http://www.comsol.com. 
[96] R. Ingram. 2011. A Mixed Finite Element Approximation of Stokes- Brinkman and NSBrinkman Equation for NonDarcian Flows. SIAM J. Numer. Anal. 49: 491-520.

[97] P. Angot. 1999. Analysis of Singular Perturbations on the Brinkman Problem for Fictitious Domain Models of Viscous Flows. Math. Methods Appl. Sci. 22: 1395-1412.

[98] Ansys CFX. 2013. CFX-Solver: Theory.

[99] N. Shamsuddin, D. B. Das, and V. M. Starov. 2015. Filtration of Natural Organic Matter using Ultrafiltration Membranes for Drinking Water Purposes: Circular Crossflow Compared with Stirred Dead-end Flow. Chem. Eng. J. 276: 331-339.

[100] A. Parasyris, M. Discacciati, and D. B. Das. 2019. Mathematical and Numerical Modelling of Hybrid Water Purification Systems. Technical Report. Loughborough University.
[101] A. Parasyris, M. Discacciati, and D. B. Das. 2019. Mathematical and Numerical Modelling of a Water Purification System. Proceedings of the Annual Conference of the UK Association for Computational Mechanics, City University of London, 2019. http://ukacm.org/.

[102] D. Boffi, F. Brezzi, and M. Fortin. 2013. Mixed Finite Element Methods and Applications. Springer, Heidelberg,

[103] P. Hansbo and A. Szepessy. 1990. A Velocity-pressure Streamline Diffusion Finite Element Method for the Incompressible NavierStokes Equations. Comput. Methods Appl. Mech. Eng. 84(2): 175-192.

[104] J. Sogn. 2014. Stabilized Finite Element Methods for the Brinkman Equation on Fitted and Fictitious Domains. Master's Thesis. University of Oslo. 